\title{
Bisa ng Filtech Web Apps bilang kagamitang panturo sa asignaturang Filipino ng Lungsod ng Pasig
}

Iranzo, Rhea V. $\square$

Buting Senior High School, Division of Pasig, Philippines (epyrhea01@gmail.com)

Revised: 1 October 2020 DOI: $10.5861 /$ ijrse.2020.5924

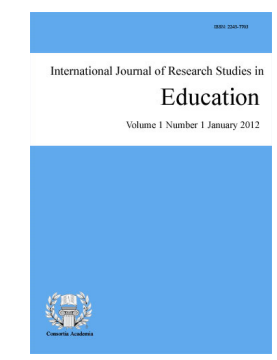

ISSN: $2243-7703$

Accepted: 25 October 2020 Online ISSN: 2243-7711

OPEN ACCESS

\section{Abstract}

This study aims to measure the effectiveness of Filtech Web Apps in teaching narrative and persuasive text in Filipino subject to Grade 11 senior high school students of Buting Senior High School. A quasi-experimental research design was employed, where student-respondents were divided into two groups - the experimental group and the comparison group. The comparison group was subjected to traditional teaching method while the experimental group used the Filtech Web Apps. The scores obtained by each group of students in pretest, posttest, formative and summative examination were computed and used as the basis to determine the effectiveness of the Filtech Web Apps. As a result of the pretesting, no significant difference was noted in the persuasive text and significant difference appeared in narrative text. Whereas the experimental group obtained higher score compared to comparison group. The academic performance of the experimental group subjected to Filtech Web Apps performed better in terms of their average scores in formative, summative and post-test evaluation, than the comparison group who underwent the traditional method of teaching. The research study was significant as it confirmed the effectiveness of using Filtech Web Apps toward the objective of improving the performance of Grade 11 students in understanding narrative and persuasive text. The study, therefore, strongly recommends the adaptation of Filtech Web Apps and the use of other appropriate modern teaching technology, particularly in teaching Filipino subject. This is to enhance the understanding, the content evaluation skill and to further motivate students to study Filipino language. Similarly, Filipino teachers are encouraged to introduce innovation in their teaching methodology, so that these groups of millennial students would find the subject and topics interesting. This would also promote and stimulate interaction, collaboration, and active participation among students.

Keywords: Filtech Web Apps; traditional teaching; teaching material; Filipino subject 


\section{Bisa ng Filtech Web Apps bilang kagamitang panturo sa asignaturang Filipino ng Lungsod ng Pasig}

\section{Panimula}

Sa mabilis na pagbabago ng panahon sa larangan ng teknolohiya, mabilis ding binago ang buhay ng bawat mag-aaral sa sangkatauhan. Isang malinaw na katotohanan ang paggamit ng iba't ibang uri ng teknolohiya na kung saan malaki ang gampanin nito sa edukasyon para malinang ang mataas na kompetensi at opurtunidad. Isa rito ng e-learning web- based module na ginagamit bilang face to face education. Ito ay nagbibigay sa mag-aaral nang makabuluhang epekto sa pagpoproseso ng kaalaman dahil sa kolaborasyon at pakikipag-interaksyon, na ginagamitan ng internet at infranet. Ayon kay (Santiago, 2011), ang gamit ng e-learning ay mabuting paraan ng paglilipat ng kaalaman mula sa guro patungo sa mga mag-aaral. Ito ang kasanayang dapat matutunan ng mga guro para sa ikakaunlad ng karunungan ng mga mag-aaral sa iba't ibang larangan. Ayon naman sa (Kagawaran ng Edukasyon ng Pasig, 2016) "Nasa edukasyon ang susi sa komportableng buhay". Makakamit lamang ito ng bawat mag-aaral sa tulong ng mga guro sa paggamit ng epektibong estratehiya sa pagtuturo ng ibat ibang asignatura tulad ng Filipino.

Sa pag-aaral ni (Cox, 2014), binanggit niya ang impormasyon ng media at teknolohiya ang pangunahing pangangailangan ng edukasyon. Sa kasulukuyan nagagamit na ang teknolohiya sa ibat- ibang sektor. Kung bibigyan ito ng pokus mabibigyan ng paglalapat ang lipunang ginagalawan ng mga mag-aaral. Nagkakaroon nang malinaw na tunguhin at mapaghamong gawain upang maging matatas ang antas na kasanayan ng mga nasa komunidad. Lalo sa proseso ng kaalaman sa larangan ng wika na mahalagang sangkap upang ang komunikasyon ng mag-aaral ay mapaunlad kailangan mapatatag ang pagiging kolaboratibo, kritikal na pag-iisip, komunikasyon at pagiging malikhain. Kaya sinubok ng pag-aaral na ito ang bisa ng pagtuturo ng asignaturang Filipino gamit ang tradisyonal at maging bisa nito sa gamit ang Filtech Web Apps.

\subsection{Layunin}

Layunin ng pag-aaral na ito na maipakita ang bisa ng FILTECH WEB APPS bilang makabagong kagamitang panturo sa asignaturang Filipino sa mga mag-aaral ng Grade 11 ng Lungsod ng Pasig. Sa pag-aaral na ito nasagot ng pag-aaral ang mga sumusunod na tanong;

$>\quad$ Ano ang resulta ng paunang pagsusulit ng mga mag-aaral ng pangkat kontrol at pangkat experimental?

$>\quad$ Ano ang resulta ng formative test ng mga mag-aaral ng pangkat eksperimental at pangkat kontrol?

A Ano ang resulta ng panapos na pagsusulit ng mga mag-aaral sa pangkat eksperimental at pangkat kontrol?

> Mayroon bang pagkakaiba ang resulta ng formative test sa pangkat ng eksperimental at pangkat kontrol?

> Mayroon bang makabuluhang pagkakaiba ang bisa ng FILTECH WEB APPS bilang experimental at pangkat kontrol sa pagtuturo ng tekstong naratibo at tekstong persweysib batay sa mga resulta ng mga sumusunod: a. Summative test, at b. Posttest?

\section{Metodolohiya}

Sa paghahangad na tuklasin ng mananaliksik ang mabisang paraan ng pagtuturo sa mga mag-aaral ng Paaralan ng Lungsod ng Pasig, taong panunuran 2018-2019 gumamit ng isang uri ng experimental na tinatawag 
na quasi-eksperimental, upang matukoy ang bisa ng paggamit ng Filtech Web Apps sa pagtuturo ng asignaturang Filipino. Ito ay kinabibilangan ng dalawang panukat: pre-posttest na dinisenyo; ang experimental at kontroladong grupo bilang partisipant ng pag-aaral.

Ang mga kalahok sa pag-aaral na ito ay mga mag-aaral ng Greyd 11 ng Buting Senior High School ng Lungsod ng Pasig sa taong-panuruan 2018-2019. Ang pagpili ng kalahok sa bawat strand ay nakabase sa may gradong 84-74 kaya hindi pare-parehas ang pagkuha ng populasyon sa bawat seksyon napili ng mananaliksik. Kinuha ang grado sa talaan ng mga guro sa unang semestre. Mula sa tradisyunal na pangkat at sa experimental na pangkat. Hindi pinili ang may mataas na grado ng mga mag-aaral sapagkat ang kanilang kasanayan sa paggamit ng teknolohiya ay sapat at may malalim na pag-unawa, kaya naman pinagtuunan ng mananaliksik ay ang mababa ang grado upang matiyak at maging malinaw ang husay ng kakalabasan paggamit ng Filtech Web Apps.

Gumawa ng liham pahintulot sa kinauukulan ng Dibisyon ng Pasig at sa punungguro ng Buting Senior High School ng Lungsod ng Pasig, upang maisakatuparan ang pag-aaral. Itinuro rin sa mga guro kung paano gamitin ang Filtech Web Apps sa pamamagitan ng LAC Session, bago ipagamit Namahagi rin ng talatanungan at nakipanayam sa mga guro at sa mga MSIT para sa validasyon ng Filtech Web Apps (Ang resulta makikita sa kabuuan ng pag-aaral na ito). Kung saan naging matagumpay ang naging tugon mula sa probisyon kaalaman, katangian ng Filtech sa guro at mag-aaral, interaksyon ng mag-aaral sa software, teknikal at porma. Samantalang sa pakikipanayam sa mga eksperto sa teknolohiya ang weighted mean 1 na nagsasabing tama ang proseso ng pagbuo ng apps na ito bagama't limitado ang paggamit kung online dahil sa kawalan ng internet connection ng mga mag-aaral.

\subsection{Instrumento ng pananaliksik}

Ginamit ng mananaliksik ang curriculum guide ng K to 12 na sinipi sa pinagyamang Pluma ng Pagbasa at Pagsusuri ng Iba't Ibang Teksto. Dalawang aralin ang inihanda para sa Kontrol at Eksperimental na Pangkat. Ang tekstong ginamit ay tekstong persweysib at tekstong naratibo. Gumamit din talahanayang ispesipikasyon ang mga pagsusulit na ginamit upang matukoy ang bisa ng Filtech Web Apps. Bago pasagutan ang mga pagsusulit nagsagawa ng dalawang beses na validasyon upang mabalido ang resulta. Mula sa resulta ng maikling pagsusulit, pangkatang gawain at isahang gawain para sa resulta ng pormatibo. Gayundin ang sumatibong pasalita at pasulat. Sa paggawa ng ng pagsusulit para pre-test at post-test ito ito ay binase sa talahanayang ispesipikasyon kung saan makikita ang kaalaman, pag-unawa at aplikasyon. Ang frequency tamang kasagutan sa bawat aytem ay nakabatay sa K-12 Curriculum Guide.

\subsection{Istatistikal na pagsusuri ng datos}

Upang matukoy ang resulta pre-test, post-test, formative test para sa pangkat eksperimental at pangkat control ang ginamit ay mean at standard deviation. Upang maihambing ang pagkakaiba ng resulta gumamit ang mananaliksik ng t-test. Gayundin para makita ang bisa ng Filtech Web Apps gumamit ng mean at t-test. Sa pagsusuri at pagtataya ng makabuluhang pagkakaiba ang unibersal na level of significance na 0.5 ang ginamit.

\section{Paglalahad ng resulta}

Inilalahad dito ang analisis at pagbibigay kahulugan sa mga nakalap na datos mula sa pauna at panapos na pagsubok ng mga mag-aaral sa dalawang pangkat na tinuruan gamit ang tradisyonal na pamamaraan at Filtech Web Apps sa pagtuturo ng kasanayang persweysib at kasanayang naratibo. Ang lahat ng resulta ay ginamitan ng ibang iba't istatistikal na pamamaraan, para maipakita ang tunay na resulta at walang pinapanigan. 
Iranzo, R. V.

\section{Talahanayan 1}

Buod na impormasyon hinggil sa resulta ng paunang pagsusulit ng dalawang grupo sa kasanayang naratibo

\begin{tabular}{|c|c|c|c|c|c|c|c|}
\hline Grupo & & Mean & Number & $S D$ & Min. & Max. & Skewness \\
\hline Tradisyonal & & 16.55 & 56 & 3.60 & 9 & 25 & -.162 \\
\hline Eksperimental & & 21.06 & 54 & 5.12 & 12 & 35 & -.331 \\
\hline & Kabuoan & 18.76 & 110 & 4.94 & 9 & 35 & .521 \\
\hline
\end{tabular}

Ang resulta ang paunang pagsusulit sa dalawang grupo sa tekstong naratibo kung pagbabatayan ang mean mas mataas ang resulta ng pangkat eksperimental kaysa sa pangkat kontrol. Gayundin ang kinalabasan ng standard deviation na malayo ang agwat ng dalawang grupo. Samantalang sa resulta ng skewness ang bunton ng mag-aaral na may mataas na iskor ay positibo para sa tradisyonal kaysa sa filtech.

Pinatunayan ito ni (Poon, 2013), mula sa kanyang pag-aaral na bleanded learning nagkakaroon ng motibasyon ang mag-aaral na matuto ng sarili at humanap ng kanyang sariling pamamaraan ayon sa kanyang oras.

\section{Talahanayan 2}

Buod na impormasyon hinggil sa resulta ng paunang pagsusulit ng dalawang grupo sa kasanayang persweysib

\begin{tabular}{|c|c|c|c|c|c|c|c|}
\hline Grupo & & Mean & Number & $S D$ & Min. & Max. & Skewness \\
\hline Tradisyonal & & 16.07 & 56 & 3.59 & 9 & 22 & -.253 \\
\hline Eksperimental & & 16.11 & 54 & 4.01 & 9 & 28 & .764 \\
\hline & Kabuoan & 16.09 & 110 & 3.79 & 9 & 28 & .328 \\
\hline
\end{tabular}

Ang resulta naman ng tekstong persweysib sa dalawang grupo kung pagbabatayan muli ang mean naging mataas pa rin ang nakuhang resulta ng filtech kaysa sa pangkat kontrol. Samantalang sa standard deviation na nagpapahiwatig pa rin ng agwat ng distribusyon ng pagsusulit nangangahulugan na normal ang distribusyon. Gayundin din ang skweness na ang bunton ng iskor ay positibo para sa eksperimental at negatibo para sa tradisyonal.

Batay sa kinalabasan ng tekstong naratibo at tekstong persweysib naging kasiya-siya ang markang nakuha ng mga mag-aaral sa paggamit ng Filtech Web Apps na. Patunay lamang ito na malaki ang ambag ng teknolohiya sa mga mag-aaral.

\subsection{Pormatib na pagsusulit ng mga mag-aaral ng pangkat eksperimental at pangkat kontrol}

\section{Talahanayan 3}

Buod na impormasyon hinggil sa resulta ng pormatib ng dalawang grupo ng kasanayang naratibo

\begin{tabular}{|c|c|c|c|c|c|c|c|}
\hline Grupo & & Mean & Number & $S D$ & Min. & Max. & Skewness \\
\hline Tradisyonal & & 40.18 & 56 & 2.41 & 36 & 46 & .596 \\
\hline Eksperimental & & 48.81 & 54 & 2.67 & 43 & 54 & -.004 \\
\hline & Kabuoan & 44.42 & 110 & 5.02 & 36 & 54 & .124 \\
\hline
\end{tabular}

Sa naging resulta ng paghahambing na mean value sa tekstong naratibo, malinaw na makikita ang pagkakaiba ng marka. Higit na kapansin-pansin pa rin dito ang mas mataas na bilang na naitala na ang mga mag-aaral na gumamit ng filtech web apps. Ang standard deviation ay nagpapahiwatig naman ng halos magkalapit na iskor ng mga mag-aaral sa dalawang grupo ng kanilang pagsusulit. Ipinakikita naman ng skewness ng bunton o pagkakapangkat na iskor na naitala ng bawat grupo. Para sa tradisyonal nagpapakita ng positibo at negatibo para sa eksperimental. 
Bisa ng Filtech Web Apps bilang kagamitang panturo sa asignaturang Filipino ng Lungsod ng Pasig

Talahanayan 4

Buod na impormasyon hinggil sa resulta ng pormatib ng dalawang grupo sa kasanayang persweysib

\begin{tabular}{|c|c|c|c|c|c|c|c|}
\hline Grupo & & Mean & Number & $S D$ & Min. & Max. & Skewness \\
\hline Tradisyonal & & 40.25 & 56 & 3.28 & 34 & 49 & .191 \\
\hline Eksperimental & & 48.70 & 54 & 3.80 & 39 & 57 & -.216 \\
\hline & Kabuoan & 44.40 & 110 & 5.52 & 34 & 57 & .142 \\
\hline
\end{tabular}

Sa naging resulta ng paghahambing na mean value sa tekstong persweysib, malinaw na makikita ang pagkakaiba ng marka. Higit na kapansin-pansin pa rin dito ang mas mataas na bilang na naitala na ang mga mag-aaral na gumamit ng filtech web apps.

Ang standard deviation ay nagpapahiwatig naman ng halos magkalapit na iskor ng mga mag-aaral sa dalawang grupo ng kanilang pagsusulit. Nangangahulugan ito na ang bilang ng mga mag-aaral na may mababang iskor ay medyo hindi malayo sa bilang ng mag-aaral na may mataas na iskor. Ipinakikita naman ng skewness ng bunton o pagkakapangkat na iskor na naitala ng bawat grupo. Para sa tradisyonal nagpapakita ng positibo at negatibo para sa eksperimental.

Batay sa resulta ng pagsusulit ng pormatibong pagsusulit ng dalawang grupo sa pagtuturo ng tekstong naratibo at persweysib naging lubhang naging kasiya siya ang resulta ng nakuhang marka ng mga mag-aaral na kung saan muling napatunayan ang bisa ng paggamit ng Filtech Web Apps kaysa sa tradisyonal. Sinasabi nga ni (Davis, 2015), mahalaga ang pagkakaroon ng pormatibong pagsusulit para makita ang husay ng pagtuturo ng guro dahil ito ang nagpapakita ng akademikong performans ng mga mag-aaral.

\section{Talahanayan 5}

Buod na impormasyon hinggil sa resulta ng sumatibo (unit) na pagsusulit ng dalawang grupo

\begin{tabular}{|c|c|c|c|c|c|c|c|}
\hline Grup & & Mean & Number & $S D$ & Min. & Max. & Skewness \\
\hline Tradisyonal & & 20.29 & 56 & 2.92 & 12 & 28 & -.325 \\
\hline Eksperimental & & 24.56 & 54 & 2.16 & 20 & 29 & -.061 \\
\hline & Kabuoan & 22.38 & 110 & 3.34 & 12 & 29 & -.441 \\
\hline
\end{tabular}

Sa naging resulta ng paghahambing na mean value sa sumatibong pagsusulit, kapansin-pansin pa rin dito ang mas mataas na bilang na naitala na ang mga mag-aaral na gumamit ng filtech web apps. Ang standard deviation ay nagpapahiwatig naman ng halos magkalapit na iskor ng mga mag-aaral sa dalawang grupo ng kanilang pagsusulit. Nangangahulugan ito na ang bilang ng mga mag-aaral na may mababang iskor ay medyo hindi malayo sa bilang ng mag-aaral na may mataas na iskor ng tradisyunal. Ipinakikita naman ng skewness ng bunton o pagkakapangkat na iskor na naitala ng bawat grupo. Nagpapakita ang tradisyonal ng positibo habang negatibo para sa eksperimental. Dahil tumaas ang bunton ng mataas na iskor sa pangkat tradisyonal.

Patunay ang kinalabasan mula sa pag-aaral ni (Balquin, 2017), na sinasabing lumalabas na angkop sa kakayahan at kawilihan ng mga mag-aaral ang alternatibong pamamaraan. Naakit ito sa aktibong partisipasyon ng mga mag-aaral kung kaya't nahikayat niya ang mga mag-aaral na masagot ang mga pagsasanay ng buong husay at may kawastuhan.

Ibig lamang ipahiwatig na nasa guro pa rin ang ikapagtatagumpay ng pagkatuto ng mga mag-aaral.

\section{Talahanayan 6}

Buod na impormasyon hinggil sa resulta ng sumatibong pagsusulit na pasalita ng dalawang grupo

\begin{tabular}{|c|c|c|c|c|c|c|c|}
\hline Grupo & & Mean & Number & $S D$ & Min. & Max. & Skewness \\
\hline Tradisyonal & & 12.70 & 56 & 1.73 & 9 & 16 & -.236 \\
\hline Eksperimental & & 15.15 & 54 & 1.25 & 13 & 18 & -.913 \\
\hline & Kabuoan & 13.90 & 110 & 1.94 & 9 & 18 & -.315 \\
\hline
\end{tabular}

Sa naging resulta ng paghahambing na mean value ng sumatibong pagsusulit na pasalita, malinaw pa rin ang 
pagkakaiba ng marka. Ang mataas na bilang na naitala na ang mga mag-aaral na gumamit ng filtech web apps Ang standard deviation ay nagpapahiwatig naman ng halos magkalapit na iskor ng mga mag-aaral sa dalawang grupo nangangahulugan ito na ang bilang ng mga mag-aaral na may mababang iskor ay medyo hindi malayo sa bilang ng mag-aaral na may mataas na iskor. Ipinakikita naman ng skewness ng bunton o pagkakapangkat na iskor na naitala ng bawat grupo. Nagpapakita ang tradisyonal ng negatibo at positibo para sa eksperimental.

Batay naman sa kinalabasan ng sumatibong pagsusulit at sumatibong pagsusulit na pasalita naging kasiya siyang muli ang resulta ng pagsusulit.

\section{Talahanayan 7}

Buod na impormasyon hinggil sa resulta ng panapos na pagsusulit ng dalawang grupo sa kasanayang naratibo

\begin{tabular}{|c|c|c|c|c|c|c|c|}
\hline Grupo & & Mean & Number & $S D$ & Min. & Max. & Skewness \\
\hline Tradisyonal & & 22.20 & 56 & 3.32 & 12 & 30 & -.271 \\
\hline Eksperimental & & 27.76 & 54 & 3.90 & 20 & 37 & .008 \\
\hline & Kabuoan & 24.93 & 110 & 4.56 & 12 & 37 & .144 \\
\hline
\end{tabular}

Sa naging resulta ng panapos na pagsusulit sa kasanayang naratibo makikita na mean value, kapansin-pansin pa rin dito ang mas mataas na bilang na naitala na ang mga mag-aaral na gumamit ng filtech web apps Ang standard deviation ay nagpapahiwatig naman ng halos magkalapit na iskor ng mga mag-aaral sa dalawang grupo ng kanilang pagsusulit. Ipinakikita naman ng skewness ng bunton o pagkakapangkat na iskor na naitala ng bawat grupo. Muling nagpapakita ang tradisyonal ng negatibo at positibo para sa eksperimental.

\section{Talahanayan 8}

Buod na impormasyon hinggil sa panapos na pagsusulit ng dalawang grupo sa kasanayang persweysib

\begin{tabular}{|c|c|c|c|c|c|c|c|}
\hline Grupc & & Mean & Number & $S D$ & Min. & Max. & Skewness \\
\hline Tradisyonal & & 20.12 & 56 & 2.62 & 13 & 25 & -.380 \\
\hline Eksperimental & & 23.04 & 54 & 3.23 & 13 & 29 & -.870 \\
\hline & Kabuoan & 24.55 & 110 & 3.27 & 13 & 29 & -.270 \\
\hline
\end{tabular}

Sa naging resulta naman ng panapos na pagsusulit ng dalawang grupo sa kasanayang persweysib ang mean value malinaw na makikita ang pagkakaiba ng marka. Kapansin-pansin pa rin dito ang mas mataas na bilang na naitala na ang mga mag-aaral na gumamit ng filtech web apps. Ang standard deviation ay nangangahulugan ng bilang ng mga mag-aaral na may mababang iskor ay medyo malapit sa bilang ng mag-aaral na may mataas na iskor. Ipinakikita naman ng skewness ng bunton o pagkakapangkat na iskor na naitala ng bawat grupo. Muling nagpapakita ang tradisyonal ng negatibo at positibo para sa eksperimental.

Muling ipinakikita ang pagkakaron ng pagkakaiba ang resulta ng dalawang paraan ng pagtuturo na kung saan nagpakita ng muling pagtaas ng resulta sa pagsusulit na panapos.

\subsection{Pagkakaiba ang resulta ng formative test sa pangkat ng eksperimental at pangkat kontrol}

\section{Talahanayan 9}

Makabuluhang pagkakaiba ng performans ng dalawang grupo ng pormatib

\begin{tabular}{lccccc}
\hline \multicolumn{1}{c}{ Kasanayan } & Mean difference & $d f$ & $t$-value & effect size & aklsyon/desisyon \\
\hline Naratibo & -8.64 & 108 & $-17.81^{* *}$ & 3.39 & May makabuluhang pagkakaiba/Reject Ho \\
Persweysib & -8.45 & 108 & $-12.52^{* *}$ & 2.38 & May makabuluhang pagkakaiba/Reject Ho \\
\hline Note. ${ }^{* * h i g h l y}$ significant with $p<$.01. Cohen's d: .20 - small, $.50-$ medium, $.80-$ large. &
\end{tabular}

Ipinakikita sa datos nasa talahanayan 9 na may makabuluhang pagkakaiba ang performans ng dalawang grupo sa pormatib. Ang naratibo at persweysib ay hindi nagkakalayo ang mean difference at ang agwat ng t-value na nangangahulugang may malaking bisa ang ginamit na Filtech sa performans ng mga mag-aaral sapagkat malaki ang naging effect size ng pormatibong pagsusulit sa pangkat tradisyonal at eksperimental na pangkat. Tinugunan ito ng pag-aaral nina (Kaya at Balta, 2016), ang paggamit ng Socrative, isang uri ng edtec

92 Consortia Academia Publishing (A partner of Network of Professional Researchers and Educators) 
Bisa ng Filtech Web Apps bilang kagamitang panturo sa asignaturang Filipino ng Lungsod ng Pasig

dahil nagbibigay ito ng motibasyon ang mga-aaral na ito ang gamitin sa kanilang pag-aaral dahil kahit saan makapag-aaral ng advance. Ang socrative na ito ay kahintulad ng Filtech Web Apps kaya ito ang patunay na hindi dapat iwasan ang pagiging inobatibong guro.

\subsection{Makabuluhang pagkakaiba ng bisa ng Filtech Web Apps bilang eksperimental at pangkat kontrol sa} pagtuturo ng tekstong naratibo at tektong perwseysib batay sa sumatibo at post test

\section{Talahanayan 10}

Makabuluhang pagkakaiba ng performans ng dalawang grupo ng sumatibong pagsusulit

\begin{tabular}{|c|c|c|c|c|c|}
\hline Kasanayan & Mean difference & $d f$ & $t$-value & effect size & aklsyon/desisyon \\
\hline $\begin{array}{l}\text { Traditional vs } \\
\text { eksperimental }\end{array}$ & -4.27 & 108 & $-8.69 * *$ & 1.66 & May makabuluhang pagkakaiba/Reject Ho \\
\hline
\end{tabular}

Ipinakikita ng nasa talahanayan 10 ang makabuluhang pagkakaiba ng performans ng dalawang grupo sa sumatibong pagsusulit. Makikita sa talahanayan ang naging makabuluhang pagkakaiba ng performans ng dalawang grupo sa sumatibong pagsusulit na may mean difference na apat at dalawamput pito na puntos na may malaking effect size patunay na may malaking makabuluhang pagkakaiba ang naging naging performans ng mga mag-aaral sa sumatibong pagsusulit.

\section{Talahanayan 11}

Makabuluhang pagkakaiba ng performans ng dalawang grupo sa sumatibo (oral)

\begin{tabular}{|c|c|c|c|c|c|}
\hline Kasanayan & Mean difference & $d f$ & $t$-value & effect size & aklsyon/desisyon \\
\hline $\begin{array}{l}\text { Traditional vs } \\
\text { eksperimental }\end{array}$ & 2.45 & 108 & $-8.51 * *$ & 1.62 & May makabuluhang pagkakaiba/Reject Ho \\
\hline
\end{tabular}

Ipinakikita ng nasa talahanayan 11 ang makabuluhang pagkakaiba ng performans ng dalawang grupo sa sumatibong pagsusulit na pasalita. Sa resulta ng sumatibong pagsusulit ng dalawang grupo ay nagpakita ng makabuluhang pagkakaiba na kung saan may mean difference na dalawa at apatnapu't lima ang tradisyonal at eksperimental na kasanayan at may malaking effect size na nangangahulugang may makabuluhang pagkakaiba ang naging performans ng dalawang grupo. Pinatunayan ito ni (Ellis, 2013), ang susi upang mapaunlad ang pormatibo at sumatibong pagtataya ay ang paghahatid ng mga kaalaman at impormasyon nang harapan.

\section{Talahanayan 12}

Makabuluhang pagkakaiba ng performans ng dalawang grupo ng pre-test

\begin{tabular}{lccccc}
\hline \multicolumn{1}{c}{ Kasanayan } & Mean difference & $d f$ & $t$-value & effect size & aklsyon/desisyon \\
\hline Naratibo & -4.50 & 108 & $-5.35^{* *}$ & 1.02 & May makabuluhang pagkakaiba \\
Persweysib & 0.04 & 108 & $0.55^{\mathrm{NS}}$ & 0.01 & Walang makabuluhang pagkakaiba/Reject Ho \\
\hline
\end{tabular}

Note. ${ }^{* *}$ highly significant with $p<.01$. Cohen's d: .20 - small, .50 - medium, .80 - large. NS - Not significant.

Ipinakikita ng nasa talahanayan 12 ang makabuluhang pagkakaiba ng performans ng dalawang grupo sa pre-test. Ang isinagawang pre-test para sa dalawang grupo ay nagpapakita ng magkaibang resulta batay sa mean difference ng naratibo at persweysib. Ang pre-test sa naratibo ay mas mataas ang mean na nangangahulugang may malaking pagkakaiba ang resulta ng pre-test kumpara sa persweysib na walang naging makabuluhang pagkakaiba ang naging resulta sa isinagawang pre-test ng dalawang grupo.

Sa puntong ito pinatutunayan ng dalawang grupo na nagsimula sa pag-aaral na nasa parehong katayuan. Nangangahulugan ito na anumang pagkakaiba sa performans ng dalawang grupo ay masasabing bunga ng pagkakaiba sa ginagamit na paraan o estratehiya. Napatunayan rin ito sa pag-aaral nina (Spector et al., 2015), ang paggamit ng teknolohiya sa mga $21^{\text {st }}$ mag-aaral ay nagiging epektibo dahil matatawag silang digital literacy at mayroon critical thinking. Lumabas sa pag-aaral na epektibo ang pagsusulit ng mga mag-aaral gamit ang iba't 
Iranzo, R. V.

ibang Edtech Binigyan nila ito ng rekomendasyon na dapat ang mga administrator ng edukasyon ay maginvest ng para sa makabagong gamitin angkop sa mag-aaral.

\section{Talahanayan 13}

Makabuluhang pagkakaiba ng performans ng grupong kontrol sa kanilang pre-test at post-test

\begin{tabular}{lccccc}
\hline \multicolumn{1}{c}{ Kasanayan } & Mean difference & $d f$ & $t$-value & effect size & aklsyon/desisyon \\
\hline Naratibo & -5.64 & 108 & $-8.62 * *$ & 1.65 & May makabuluhang pagkakaiba/Reject Ho \\
Persweysib & -4.05 & 108 & $-6.21 * *$ & 1.29 & May makabuluhang pagkakaiba/Reject Ho \\
\hline Note. ${ }^{* * h i g h l y ~ s i g n i f i c a n t ~ w i t h ~} p<.01$. Cohen's d: .20 - small, .50- medium, .80 - large.
\end{tabular}

Ipinakikita ng talahanayan 13 ang makabuluhang pagkakaiba ng performans ng grupong kontrol sa kanilang pre-test at post-test. Sa nakuhang resulta ng grupong kontrol sa pre-test at post-test ay halos di naglalayo ang mean difference ng naratibo at persweysib maging ang t-value na may malaking effect size na nangangahulugang parehas ay may malaking makabuluhang pagkakaiba ang naging performans ng mga mag-aaral sa isinagawang pre-test at post-test na ginamitan ng Filtech application. Sa bahaging ito makikita na ang grupong tradisyonal ay nagtamo rin ng pag-unlad sa kanilang pag-aaral. Ibig lamang sabihin na hindi lng sa mga guro o estratehiyang kanyang ginagamit nakasalalay ang pagkatuto ng mga mag-aaral kundi paano gagamitin ng guro ang estratehiyang makabago o tradisyonal, para sa ikakatagumpay ng pagtuturo ng guro sa pagkatutuo ng mga mag-aaral.

\section{Talahanayan 14}

Makabuluhang pagkakaiba ng performans ng grupong experimental sa kanilang pre-test at post-test

\begin{tabular}{lccccc}
\hline \multicolumn{1}{c}{ Kasanayan } & Mean difference & $d f$ & $t$-value & effect size & aklsyon/desisyon \\
\hline Naratibo & -6.15 & 106 & $-6.73 * *$ & 1.47 & May makabuluhang pagkakaiba/Reject Ho \\
Persweysib & -6.35 & 106 & $-8.44 * *$ & 1.90 & May makabuluhang pagkakaiba/Reject Ho \\
\hline Note **highly significant with $p<01$ Cohen's d: 20 & - small, 50 - medium, $80-$ large.
\end{tabular}

Note. **highly significant with $p<.01$. Cohen's d: $.20-$ small, .50 - medium, $.80-$ large.

Ang talahanayan 21 ay nagpapakita ng makabuluhang pagkakaiba ng performans ng grupong eksperimental sa kanilang pre-test at post test. Maliwanag na ipinahihiwatig dito performans ng grupong eksperimental sa kasanayang naratibo at persweysib. Malinaw na ipinakikita sa talahanayan dalawampu na halos hindi rin naglalayo ang mean difference, $t$-value at malaking effect size sa naging performans ng grupong eksperimental sa kanilang pre-test at post-test mula sa naratibo at persweysib na kasanayan. Ang resultang ito ay nangangahulugang parehong may makabuluhang pagkakaiba ang naging performans ng mga mag-aaral gamit ang Fil Tech Apps sa isinagawang pagsusulit.

\section{Talahanayan 15}

Makabuluhang pagkakaiba ng performans sa pretest at post-test ng dalawang grupo

\begin{tabular}{lccccc}
\hline \multicolumn{1}{c}{ Kasanayan } & Mean difference & $d f$ & $t$-value & effect size & aklsyon/desisyon \\
\hline Naratibo & -5.56 & 108 & $-8.06^{* *}$ & 1.31 & May makabuluhang pagkakaiba/Reject Ho \\
Persweysib & -2.91 & 108 & $-5.19^{* *}$ & 1.64 & May makabuluhang pagkakaiba/Reject Ho \\
\hline Note. $* *$ highly significant with $p<.01$. Cohen’s d: .20 & - small, .50 - medium, .80 - large.
\end{tabular}

Ang talahanayan 15 ay nagpapakita ng makabuluhang pagkakaiba ng performans ng dalawang grupo sa pre-test at post-test. Sa puntong ito sinasabi ng hayagang ang malaking pagkakaiba sa kasanayang naratibo sa grupong eksperimental kumpara sa grupong tradisyonal batay sa kinalabasan ng test statistics na tunay na highly significant ang pagkakaiba ng mga kasanayan. Patunay lamang ang pananaliksik ni (Celenia, 2010), kung saan siya ay gumamit ng iba't ibang estratehiya gaya ng direct teaching at vocabulary strategies. Isa sa ang Filtech Wep Apps ang metodolohiyang makakatulong sa pagkatuto ng mag-aaral, patunay ang resulta ng panapos na pagsusulit na ito.

\section{Konklusyon at rekomendasyon}

Sa pamamagitan ng mga inilahad na pagbubuo, nabuo ang sumusunod na kongklusyon.

94 Consortia Academia Publishing (A partner of Network of Professional Researchers and Educators) 
> Walang pagkakaiba ang kinalabasan ng pagsusulit sa tekstong persweysib sa paunang pagsusulit ng pangkat kontrol at pangkat eksperimental kaya't ito ay tinatanggap. Samantalang sa tekstong naratibo ay may pagkakaiba ang resulta ng dalawang grupo. Higit na nagkaroon ng mataas na iskors ang pangkat eksperimental kaya hindi tinatanggap ang hinuha.

> May pagkakaiba ang resulta ng pagsusulit ng pormatibo sa pagtuturo ng dalawang teksto, naging lubhang kasiya siya ang resulta ng Filtech Web apps kaysa sa pangkat kontrol, kaya't hindi tinatanggap ang hinuha.

> Walang makabuluhang pagkakaiba ang resulta ng akademikong performans na sumailalim sa tradisyunal na istratehiya at grupo ng gumamit ng Filtech Web Apps para sa pagtuturo ng tekstong naratibo at tekstong persweysib kaya pinasusubalian ang hinuha, batay sa resulta ng post-test at sumatibo, naging matagumpay ang ginawang pagsasaliksik dahil sa paggamit ng filtech web apps ay naging daan upang mapaunlad ang kakayahan ng mga mag-aaral sa Grade 11 sa kanilang pag-unawa sa tekstong naratibo at tekstong persweysib.

Kaya iminumungkahi ng pananaliksik na ito ang mga sumusunod:

$>$ Ang mga namumuno sa paaralan ay maaaring bigyang suporta ang paggamit ng makabagong pamamaraan sa pagtuturo ng guro, partikular sa asignaturang Filipino upang sa gayon higit silang mahasa sa pag-unawa, pagsusuri sa nilalaman ng aralin, at maganyak sa pag-aaral ng wikang Filipino.

$>$ Ang mga guro ng Filipino ay hinihimok na huwag maging kontento sa estratehiyang ginagamit sa pagtuturo. Maging inobatibo sa pagpili ng gagamiting materyal upang sa gayon maging interkatibo, kolaboratibo at aktibo ang mga mag-aaral para sa mas malalim na pag-unawa sa bawat araling itinuturo.

$>$ Ang mga mag-aaral ay hinihikayat na anumang estratehiyang gamitin sa pagtuturo ng Filipino sikaping maging matiyaga sa paghahasa ng kakayahan, maging masigasig, at kapaki-pakinabang sa talakayan.

$>$ Ang mga susunod na mananaliksik ay hinihikayat na huwag huminto sa pagtuklas ng mga makabagong estratehiya sa pagtuturo lalo na sa paggamit ng teknolohiya. Ipagpatuloy ang pag-aaral na ito para sa pagtuklas pa ng marami at higit pang kaugnayan ng mga datos na maaaring ikakaunlad pa ng pananaliksik na ito.

\section{References}

Balquin, A. (2017). Alternatibong pamamaraan ng pagtuturo ng panitikan sa pagkakatuto ng mga mag-aaral [Unpublished master thesis]. Roosevelt College, Cainta.

Celenia. (2013). The effect of the relationship between learning and teaching strategies on academic achievement. Navotas, Royal.

Cox, C. (2014). $21^{\text {st }}$ century skills and principles of flow in the foreign language classroom. Bringham Young University-Provo.

Davis, M., R. (2015). Technology fed growth in formative assessment. Education Week. http://www.edweek.org/ew/articles/2015/11/11/technology-fed-

DepEd. (2016). Education the key to a healthy life [Edukasyon ang susi sa maayos na buhay]. Retrieved from https://www.deped.gov.ph/2016/11/06/deped-edukasyon-ang-susi-sa-maayos-na-buhay/

Ellis, C. (2013). Broadening the scope and increasing usefulness of learning analytic: The case for assessment analytics. British Journal of Educational Technology, 44(4). https://doi.org/10.1111/bjet.12028

Kaya, A., \& Balta, N. (2016). Taking advantages of technologies: Using socrative in English language teaching classes. International Journal of Social Sciences and Educational Studies, 2(3), 4-12. 
Iranzo, R. V.

Poon, J. (2013). Blended learning: An institutional approach for enhancing students' learning experiences. Journal of Online Learning and Teaching, 9(2), 271-289.

Santiago, A. (2011). GENYO e-learning. What are you allabout? Retrieved from http://itswyza.wordpress.com/2011

Spector, J. M., Ifenthaler, D., Sampson, D., Yang, L., Mukama, E., Warusavitarana, A., Dona, K. L., Eichhorn, K., Fluck, A., Huang, R., Bridges, S., Lu, J., Ren, Y., Gui, X., Deneen, C. C., San Diego, J., \& Gibso, D. C. (2016). Formative assessment for 21st century learning. Educational Technology \& Society, 19(3), $58-71$.

96 Consortia Academia Publishing (A partner of Network of Professional Researchers and Educators) 